Im „Journal Club“ werden Originalarbeiten aus der internationalen Fachliteratur referiert und kommentiert.

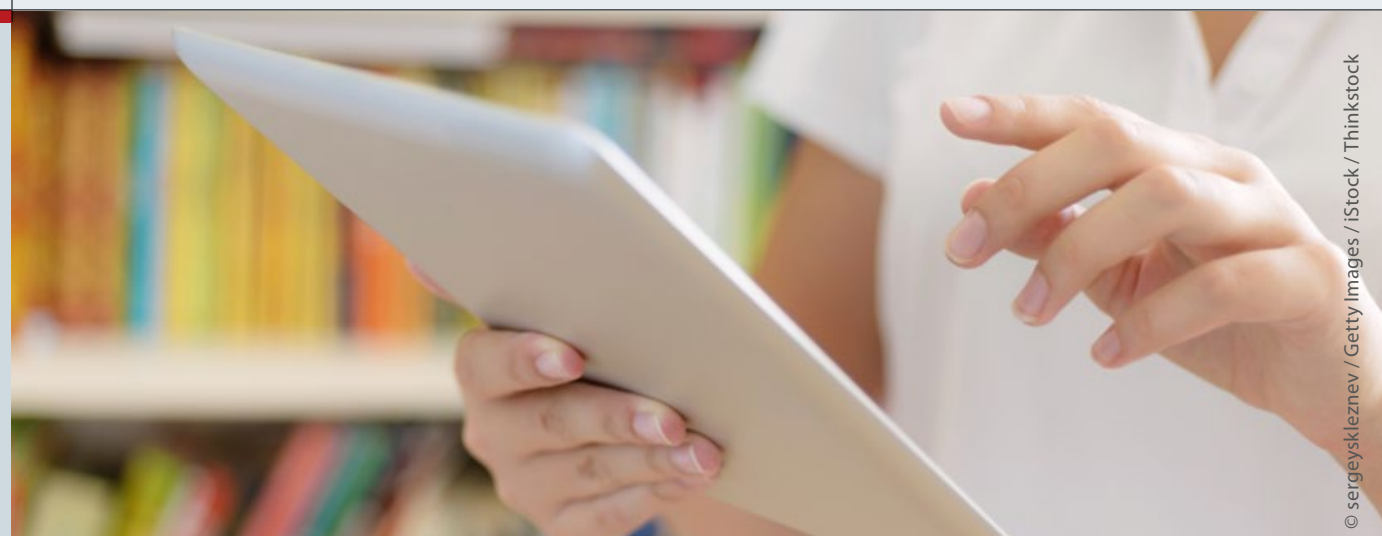

\section{Beste Pflege für zarte Babyhaut}

\begin{abstract}
Junge Mütter wollen alles richtig machen, doch fehlt ihnen oft die Erfahrung. Meist mangelt es nicht an gut gemeinten Ratschlägen von allen Seiten, diese fördern die Verunsicherung oft zusätzlich. Zusammen mit anderen europäischen Kollegen haben Dermatologen der Berliner Charité aktuelle Tipps zur Pflege der Säuglingshaut erarbeitet.
\end{abstract}

m Rahmen eines zweiten RoundtableMeetings hat Ulrike Blume-Peytavi von der Charité mit weiteren Hautexperten aus verschiedenen europäischen Ländern die Empfehlungen zur Pflege der Säuglingshaut von 2009 auf der Ba-

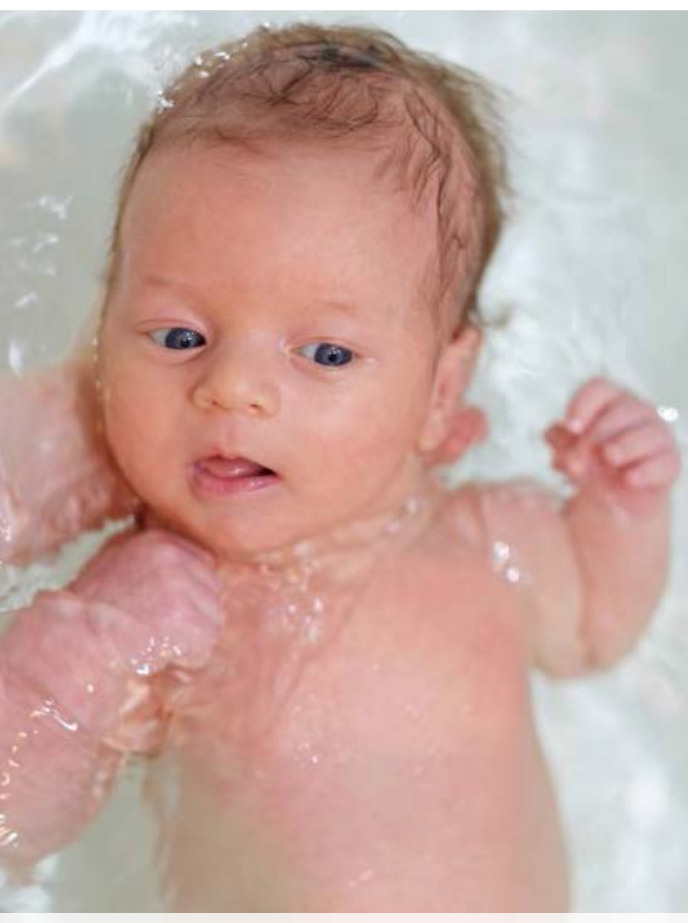

Damit die Hautreifung nicht beeinflusst wird, soll das Badewasser keine Zusätze oder nur speziell für die Babyhaut entwickelte flüssige Reinigungspräparate enthalten. sis neuer Studiendaten überprüft und auf den neuesten Stand gebracht.

\section{Baden mit oder ohne Zusatz}

Direkt nach der Geburt sollen Neugeborene nicht gewaschen, sondern möglichst vorsichtig mit einem trockenen Handtuch abgetupft werden. Sobald sich die Körpertemperatur stabilisiert hat, ist ein allabendliches Bad (mindestens aber zweibis dreimal pro Woche) über fünf bis zehn Minuten empfehlenswert, auch wenn der Nabelschnurrest noch nicht abgefallen ist. Das Baden wird ausdrücklich dem Waschen vorgezogen. Damit der Prozess der Hautreifung nicht beeinflusst wird, soll das Badewasser entweder gar keine Zusätze oder nur speziell für die Babyhaut entwickelte flüssige Reinigungspräparate enthalten. In kleinen Mengen kann auch ein Babyöl zugesetzt werden. Produkte zur Pflege von Säuglingen sollen generell eine gewisse Pufferwirkung haben, so dass sie den $\mathrm{pH}$-Wert der Haut im leicht Sauren bei etwa 5,5 halten können. Formulierungen mit aggressiven oberflächenaktiven Inhaltsstoffen wie Natriumlaurylsulfat sind zu vermeiden. Badewanne und Badespielzeug sollen nach den neuen Empfehlungen beim Bad zuhause nicht mehr desinfiziert, sondern lediglich sauber gehalten werden. Eine weitere Änderung betrifft die Raumtemperatur: Hier werden nicht mehr wie bisher $21-22^{\circ} \mathrm{C}$, sondern neuerdings bis zu $24^{\circ} \mathrm{C}$ empfohlen.

\section{Emollienzien zur Pflege}

Unmittelbar nach dem Bad soll das Baby zugedeckt und trockengetupft werden. Um die Hautbarriere zu stärken, können geeignete Emollienzien dünn aufgetragen werden. Ganz besonders profitieren hiervon Kinder mit atopischer Dermatitis oder einem hohen Risiko für diese Hauterkrankung. Pflegeprodukte sollten mindestens zweimal wöchentlich aufgetragen und der Jahreszeit angepasst werden. Bei kühlen Temperaturen etwa soll der Emolliensgehalt höher als der Wassergehalt sein, in der wärmeren Jahreszeit dagegen genügt eine weniger reichhaltige Pflege. Auch Babyöl kann vorübergehende Hauttrockenheit mildern.

Während des Zahnens muss besonders darauf geachtet werden, dass die Mundpartie durch den Speichel nicht gereizt wird. Generell sollte jede Hautreizung oder -veränderung dem Kinderarzt vorgestellt werden, um beispielsweise eine atopische Dermatitis früh zu erkennen und adäquat zu versorgen. Besteht eine neonatale oder infantile Akne, sollte auf Emollienzien verzichtet werden, um eine follikuläre Okklusion zu vermeiden.

Windeln sollen so oft wie nötig gewechselt werden, damit der Po sauber und trocken bleibt und eine Windeldermatitis vermieden wird. Von Geburt an soll der Windelbereich vorsichtig mit Wasser und Wattebäuschen oder Tupfern aus Baumwolle oder anderen für die Windelpflege entwickelten, $\mathrm{pH}$-gerechten Tüchern gereinigt und vor dem Anlegen einer neuen Windel vollständig getrocknet werden.

Blume-Peytavi $U$ et al. Recommendations from a European Roundtable Meeting on Best Practice Healthy Infant Skin Care. Pediatric Dermatology 2016; (online first) 